\title{
ESR STUDIES OF INTER- AND INTRA-LAMELLAR CATION EXCHANGE PROCESSES IN $\mathrm{Cd}_{2} \mathrm{P}_{2} \mathrm{~S}_{6}$
}

\author{
M. A. Hefni, $\dagger$ N. Nagasundaram, D. Kreszowski $\ddagger$ and A. H. Francis \\ Department of Chemistry, The University of Michigan, Ann Arbor, MI 48109-1055, U.S.A.
}

(Received 21 May 1990; accepted 25 July 1990)

\begin{abstract}
The transition metal chalcogenides, $\mathbf{M}_{2} \mathbf{P}_{2} \mathbf{S}_{6}(\mathbf{M}=$ divalent transition metal cation), are lamellar materials that undergo an unusual cation exchange process when their crystals are placed in contact with a solution of a transition metal salt. EPR spectroscopy has been used to examine the exchange of paramagnetic $\mathrm{Co}^{2+}$ for $\mathrm{Cd}^{2+}$ in diamagnetic $\mathrm{Cd}_{2} \mathrm{P}_{2} \mathrm{~S}_{6}$. It has been possible to study the uptake of ions by the lamellar lattice and to examine the cation coordination environment through its effect on the parameters of the spin-Hamiltonian. In order to extract details of the $\mathrm{Co}^{2+}$ coordination from the observed EPR spectra, theoretical values of the $g$-value have been computed as a function of several crystal field parameters. The results for $\mathrm{Co}^{2+}$ are compared with previous results for the insertion of $\mathrm{Mn}^{2+}$ into $\mathrm{Cd}_{2} \mathrm{P}_{2} \mathrm{~S}_{6}$ by cation exchange. The relative occupancy of the inter- and intra-lamellar sites is a function of the cation type and the solvent employed.
\end{abstract}

Keywords: ESR, chalcogenide, intercalation, magnetic resonance.

\section{INTRODUCTION}

The transition metal phosphorus chalcogenides $\left(\mathrm{M}_{2} \mathrm{P}_{2} \mathrm{~S}_{6}, \mathrm{M}=\right.$ transition metal) form a class of lamellar materials with crystal structures of the $\mathrm{CdCl}_{2}$ type [1]. The layers are composed of arrays of $\left[\mathrm{P}_{2} \mathrm{~S}_{6}\right]^{4-}$ units coordinated to the $\mathrm{M}^{2+}$ cations through $\mathrm{M}-\mathrm{S}$ bonds, so that the transition metal ions are in a trigonally distorted octahedral environment. The transition metal layers are separated by two planes of chalcogenide atoms, bound together by relatively weak van der Waals interactions. Because of the weak nature of these interactions, various chemical species may be intercalcated into the van der Waals gap (VWG) between the adjacent chalcogenide layers [2].

An unusual ion exchange process occurs in crystalline $\mathrm{M}_{2} \mathrm{P}_{2} \mathrm{~S}_{6}$ lattices in which the lattice cations are exchanged with intercalate cations [3]. The process is surprising in terms of the conventional view of transition metal chalcogenide lattices as covalently bonded, extended networks. In $\mathrm{Mn}_{2} \mathrm{P}_{2} \mathrm{~S}_{6}$, the $\mathrm{Mn}^{2+}$ can be exchanged at room temperature with mono- or dipositive guest cations $\left(\mathrm{G}^{+}\right.$or $\left.\mathrm{G}^{2+}\right)$ to form compounds with the composition $\mathrm{Mn}_{2-x} \mathrm{G}_{2 x / n}^{n+} \mathrm{P}_{2} \mathrm{~S}_{6}$. EXAFS studies [4] have demonstrated that $\mathrm{Ni}^{2+}$ guest cations enter the intralamellar $\mathrm{Mn}^{2+}$ vacancies in $\mathrm{Mn}_{2} \mathrm{P}_{2} \mathrm{~S}_{6}$. Recently, Clement $e t$ al. [5] studied the exchange process by systematically exchanging the lattice cation in several of the $M_{2} P_{2} S_{6}$ latticess with

† Visiting Fulbright Senior Scholar. Permanent address: Department of Physics, Faculty of Science, Assiut University. Assiut, Egypt.

$¥$ Present address: Department of Chemistry, Michigan State University, East Lansing, Michigan, U.S.A.
$\mathrm{K}^{+}$. It was found that the ease of exchange followed the sequence $\mathrm{Mn}^{2+}>\mathrm{Cd}^{2+}>\mathrm{Fe}^{2+}$ and $\mathrm{Ni}^{2+}$ showed no exchange.

Previously [6], we have used EPR to study the exchange of diamagnetic $\mathrm{Cd}^{2+}$ cations in $\mathrm{Cd}_{2} \mathrm{P}_{2} \mathrm{~S}_{6}$ by paramagnetic $\mathrm{Mn}^{2+}$. The EPR analysis demonstrated that the guest cation is partitioned between interstitial sites in the VWG and host lattice $\mathrm{Cd}^{2+}$ vacancies. Heating the intercalated lattice is required to achieve insertion from the VWG into intralamellar vacancies.

In the present work, EPR spectroscopy has been employed to examine the insertion of paramagnetic $\mathrm{Co}^{2+}$ into $\mathrm{Cd}_{2} \mathrm{P}_{2} \mathrm{~S}_{6}$. We have carried out the exchange reactions using different solvent media in order to qualitatively assess the importance of solvent coordination in the exchange process.

\section{EXPERIMENTAL}

$\mathrm{Cd}_{2} \mathrm{P}_{2} \mathrm{~S}_{6}$ was synthesized from the elements as described previously [7]. Crystals of $\mathrm{Cd}_{2} \mathrm{P}_{2} \mathrm{~S}_{6}$ containing about $1 \%$ by weight of $\mathrm{Co}^{2+}$ were obtained by the same method as for the undoped lattice, except that the required amount of dopant was also added to the reaction mixture. These crystals were used as standards for identification of intra-lamellar $\mathrm{Co}^{2+}$ residing on $\mathrm{Cd}^{2+}$ sites. In doped crystals prepared by sublimation at $>650^{\circ} \mathrm{C}$, the $\mathrm{Co}^{2+}$ cation resides exclusively on cadmium substitutional sites.

$\mathrm{Co}^{2+}$ cations were introduced into the $\mathrm{Cd}_{2} \mathrm{P}_{2} \mathrm{~S}_{6}$ lattice by a two step exchange procedure described by Clement and coworkers [5]. Large single crystals (typically $4 \times 4 \times 0.1 \mathrm{~mm}$ ) of $\mathrm{Cd}_{2} \mathrm{P}_{2} \mathrm{~S}_{6}$ were immersed in a $1 \mathrm{M} \mathrm{KCl}$ solution at room temperature for $2-3$ days. The solution was buffered to $\mathrm{pH}=7$ with 
potassium bicarbonate and was approx. $0.1 \mathrm{M}$ in EDTA. This procedure creates $\mathrm{Cd}^{2}-$ vacancies and inserts highly mobile solvated $\mathrm{K}^{+}$cations into the interstitial sites in the VWG according to the reaction,

$$
\begin{aligned}
& \mathrm{Cd}_{2} \mathrm{P}_{2} \mathrm{~S}_{6}(s)+2 x \mathrm{~K}^{+}(a q) \stackrel{\mathrm{H}_{2} \text { O EDTA }}{\longrightarrow} \\
& \mathrm{Cd}_{2-x} \mathrm{~K}_{2 x} \mathrm{P}_{2} \mathrm{~S}_{6}(s)+x \mathrm{Cd}^{2+}(a q) .
\end{aligned}
$$

The EDTA assists in the extraction of $\mathrm{Cd}^{2+}$ by forming a strong complex with the metal cation. The product crystals were immersed in $1 \mathrm{M}$ aqueous solutions of $\mathrm{CoCl}_{2}$ for 2 days in order to intercalate the host solid with $\mathrm{Co}^{2+}$ according to the following reaction

$$
\begin{aligned}
& \mathrm{Cd}_{2-x} \mathrm{~K}_{2 x} \mathrm{P}_{2} \mathrm{~S}_{6}(s)+\mathrm{Co}^{2}+\stackrel{\mathrm{H}_{2} \mathrm{O}}{\longrightarrow} \\
& \mathrm{Cd}_{2-x} \mathrm{Co}_{x} \mathrm{P}_{2} \mathrm{~S}_{6}(s)+2 x \mathrm{~K}^{+}(a q)
\end{aligned}
$$

The solution was decanted and the crystals were washed a number of times with distilled water and then air-dried.

Crystals of $\mathrm{Cd}_{2-x} \mathrm{Co}_{x} \mathrm{P}_{2} \mathrm{~S}_{6}$ were also prepared by a single step procedure used previously for the exchange of $\mathrm{Mn}^{2+}$ with $\mathrm{Cd}^{2+}$ in $\mathrm{Cd}_{2} \mathrm{P}_{2} \mathrm{~S}_{6}$. In this procedure, single crystals were immersed for 2-3 days at room temperature in a $1 \mathrm{M} \mathrm{CoCl}$ solution prepared with a solvent consisting of $80 \%$ by volume pyridine in water. The exchange may be represented as,

$$
\begin{aligned}
\mathrm{Cd}_{2} \mathrm{P}_{2} \mathrm{~S}_{6}(s)+x \mathrm{Co}^{2+} \text { (pyridine) } \longrightarrow \\
\mathrm{Cd}_{2-x} \mathrm{Co}_{x} \mathrm{P}_{2} \mathrm{~S}_{6}(s)+x \mathrm{Cd}^{2+} \text { (pyridine) } .
\end{aligned}
$$

Because the product crystals may contain intercalated solvent, it was necessary to record EPR spectra after programmed heating cycles. Programmed heat treatment of the crystals was conveniently accomplished using a DuPont Instruments Model R90 thermal analyzer equipped with a Model 951 thermogravimetric analyzer. A nitrogen purge gas was used during heat treatment to avoid oxidation of the sample. EPR spectra were measured with a Bruker ER 200E-SRC X-band spectrometer with $100-\mathrm{kHz}$ field modulation and a $T M_{110}$ cavity. The sample temperature was controlled with an Oxford Instruments Model ESR 900 continuous flow liquid helium cryostat.

\section{RESULTS}

An ESR spectrum obtained at $5 \mathrm{~K}$ from a $\mathrm{Cd}_{2} \mathrm{P}_{2} \mathrm{~S}_{6}$ crystal doped with $1 \%$ by weight of $\mathrm{Co}^{2} \pm$ during growth by vapor transport is reproduced in Fig. 1 . This spectrum is observable below about $25 \mathrm{~K}$ and is

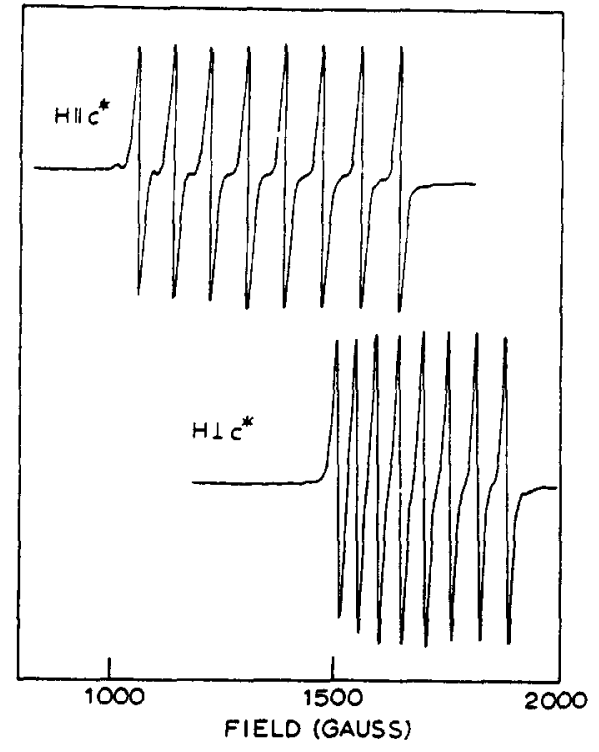

Fig. 1. The EPR spectrum of $\mathrm{Co}^{2+}$ substitutional impurity centers in $\mathrm{Cd}_{2} \mathrm{P}_{2} \mathrm{~S}_{6}$ at $T=5 \mathrm{~K}$. (a) $H$ parallel to $c^{*}$ and (b) $H$ perpendicular to $c^{*}$.

substantially independent of temperature between 20 and $4 \mathrm{~K}$ [8]. The spectrum is that of single $\mathrm{Co}^{2+}$ cations at $\mathrm{Cd}^{2+}$ vacancies. No EPR signal from pair spectra or interstitial ions was observed [9]. Experimental values of the spin-Hamiltonian parameters obtained from this spectrum are given in Table 1.

The ESR spectra shown in Fig. 2 were obtained at $5 \mathrm{~K}$ from $\mathrm{Cd}_{2-x} \mathrm{~K}_{2 x} \mathrm{P}_{2} \mathrm{~S}_{6}$ crystals exchanged with $\mathrm{Co}^{2+}$ in aqueous medium. The spectrum sharpens considerably and becomes multiple as the temperature is increased. Figure 3 illustrates the appearance of the spectrum of this material at $10 \mathrm{~K}$. A new,

\begin{tabular}{|c|c|c|c|c|c|}
\hline \multirow{2}{*}{$\begin{array}{l}\text { Compound } \\
\text { (reference) }\end{array}$} & \multirow[b]{2}{*}{$\boldsymbol{g}_{\|}$} & \multirow[b]{2}{*}{$g_{\perp}$} & $\mathbf{A}_{\|}$ & $A_{1}$ & \multirow[b]{2}{*}{ Site } \\
\hline & & & \multicolumn{2}{|c|}{$\times 10^{-4} \mathrm{~cm}^{-1}$} & \\
\hline KFS' & 5.80 & 3.44 & 184 & 47 & Trig. comp. \\
\hline $\mathrm{Cd}_{2} \mathrm{P}_{2} \mathrm{~S}_{6} \ddagger$ & 4.94 & 3.99 & 183 & 97 & Trig. \\
\hline $\mathrm{Cd}_{2} \mathrm{P}_{2} \mathrm{~S}_{6} \S$ & 4.89 & 3.94 & 189 & 103 & Trig. \\
\hline & 5.00 & 3.94 & 199 & 118 & Trig. \\
\hline $\mathrm{MgO}$ & 4.28 & 4.28 & & & Octahedral \\
\hline $\mathrm{CaO}+t$ & 4.37 & 4.37 & & & Octahedral \\
\hline $\mathrm{Cd}_{2} \mathrm{P}_{2} \mathrm{~S}_{6+\ddagger}$ & 2.21 & 4.59 & & & Trig. elong. \\
\hline $\mathrm{CdBr}_{2} 85$ & 3.76 & 4.67 & 50 & 150 & Trig. elong. \\
\hline $\mathrm{CdCl}_{2}, 8 \mathrm{~S}$ & 3.06 & 4.69 & 36 & 170 & Trig. elong. \\
\hline $\mathrm{MgCl}_{2} \$ \S$ & 2.86 & 5.05 & 45 & 161 & Trig. elong. \\
\hline
\end{tabular}
sharper spectrum appears with a slightly altered $g$-value. The temperature dependence of the sharp and broad resonances is shown in Fig. 4. The extreme

Table 1. ESR parameters for $\mathrm{Co}^{2+}$ in various coordination environments

+ In potassium fluorosilicate [10].

$¥$ Vapor doped.

Sharp spectrum.

Broad spectrum.

T [17].

$++[18]$

$¥ ¥$ Calculated, this work

$\$[9]$. 


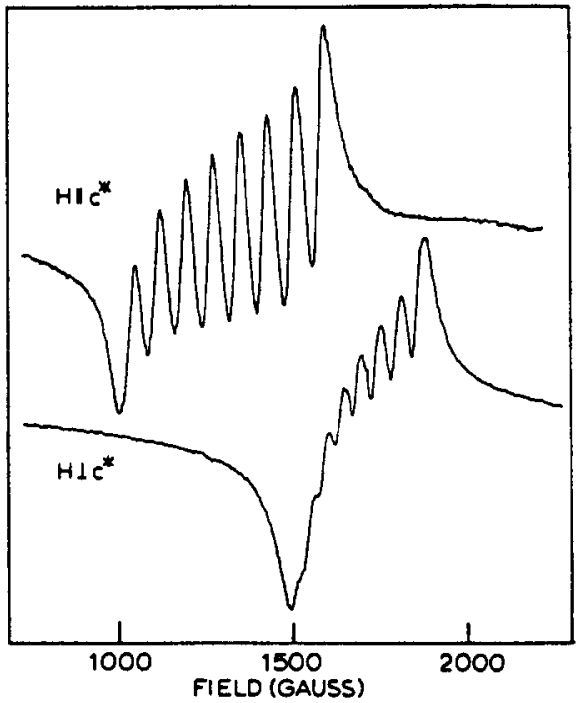

Fig. 2. The EPR spectrum of $\mathrm{Co}^{2+}$ interealated cation in $\mathrm{Cd}_{2} \mathrm{P}_{2} \mathrm{~S}_{6}$ at $T=5 \mathrm{~K}$.

temperature sensitivity of the EPR spectra of the ion exchanged lattice is markedly different from the behavior of the vapor doped lattice (Fig. 1).

The EPR spectra in Fig. 5 were obtained after briefly treating the sample crystals with a solution of $1 \mathrm{M} \mathrm{KCl}$ to replace interstitial $\mathrm{Co}^{2+}$ cations with $\mathrm{K}^{+}$. After this treatment, the spectrum became independent of temperature between 4 and $20 \mathrm{~K}$.

\section{DISCUSSION}

The EPR spectra (Figs 2 and 3) of $\mathrm{Co}^{2+}$ ions introduced into $\mathrm{Cd}_{2} \mathrm{P}_{2} \mathrm{~S}_{6}$ lattices by cation exchange are consistent with two, quite similar, cation coordination geometries. The magnitudes of the $g$-values and the $g$-value anisotropy are nearly identical and

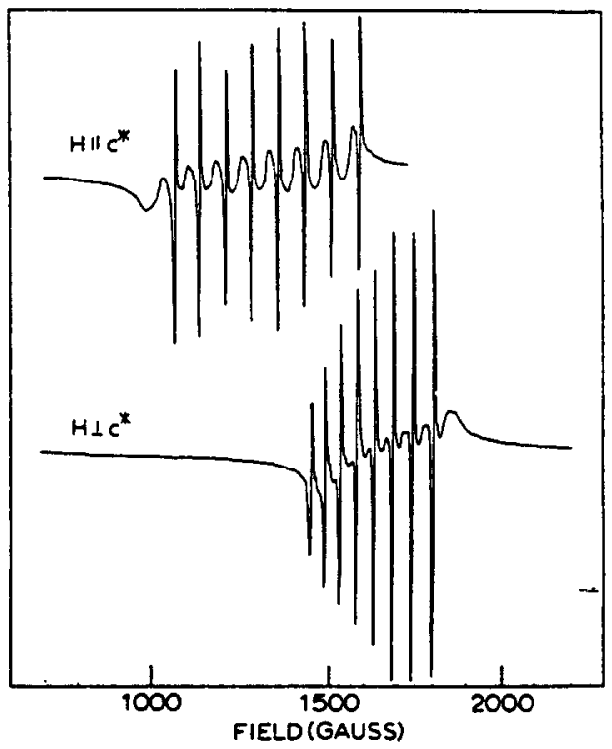

Fig. 3. The EPR spectrum of $\mathrm{Co}^{2+}$ intercalated cation in $\mathrm{Cd}_{2} \mathrm{P}_{2} \mathrm{~S}_{6}$ at $T=10 \mathrm{~K}$.

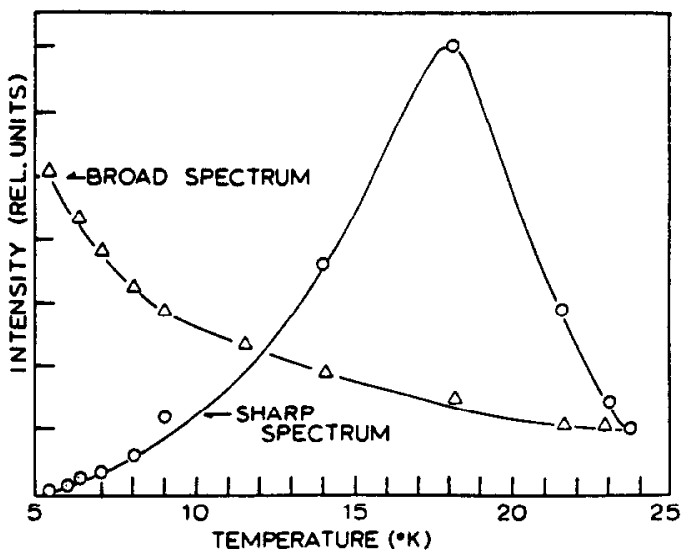

Fig. 4. Plot of the temperature dependence of the EPR site spectra obtained for $\mathrm{Co}^{2+}$ in $\mathrm{Cd}_{2} \mathrm{P}_{2} \mathrm{~S}_{6}$.

are very close to the $g$-value for substitutional $\mathrm{Co}^{2+}$ ions located at $\mathrm{Cd}^{2+}$ cation vacancies (Fig. 1). The greatest variation is observed in the value of $g_{\|}$, suggesting that differences between the sites occur principally in the $c^{*}$ direction. The linewidth and temperature dependence of the site spectra are very different, indicative of different relaxation times for each site (see Fig. 4). Heating the crystalline samples to $600^{\circ} \mathrm{C}$ to remove intercalated solvent produced no significant changes in the EPR spectra.

The angle dependences of the EPR spectra of $\mathrm{Co}^{2+}$ observed after cation exchange indicate that the ions occupy lattice sites with their spin-axes aligned with respect to the crystallographic axes. Randomly oriented ions in the VWG would produce a powder EPR spectrum with peak absorption at $g_{\perp}$. There is no evidence of a powder EPR spectrum, therefore disordered ions are either not present or are EPR silent. The oriented ions may be located at either tetrahedral or octahedral interstitial sites within

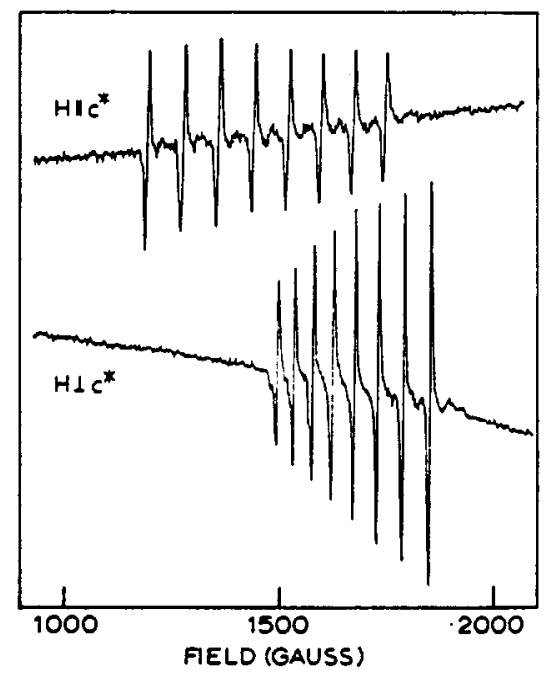

Fig. 5. The EPR spectrum of $\mathrm{Co}^{2+}$ intercalated cation $\mathrm{Cd}_{2} \mathrm{P}_{2} \mathrm{~S}_{6}$ at $5 \mathrm{~K}$ obtained after exchanging interstitial cations with $\mathrm{K}^{+}$. 
the VWG or at octahedral cadmium ion vacancies. Further details concerning the coordination of the exchanged $\mathrm{Co}^{2+}$ ions can be extracted from the magnitude and anisotropy of the components of the $g$-tensor.

\section{g-Value anisotropy and coordination environment}

In tetrahedral coordination, the crystal field splitting of the ${ }^{4} \mathrm{~F} \mathrm{Co}^{2+}$ free-ion ground term yields a ${ }^{4} A_{2 g}$ ground state. The isotropic $g$-value is given approximately by,

$$
g=g_{e}-8 \lambda / 10 D q
$$

Setting the spin-orbit coupling parameter $\lambda=-180 \mathrm{~cm}^{-1}$ and $10 \mathrm{Dq}=4300 \mathrm{~cm}^{-1}$ in eqn (1) gives $g=2.34$. No resonances are observed with $\boldsymbol{g}$-values close to that expected for tetrahedral coordination. We conclude that the tetrahedral sites in the VWG are either not occupied or are EPR silent.

In the $\mathrm{Cd}^{2+}$ sites, the ${ }^{4} F$ ground state is split by a trigonally distorted octahedral crystal field and spin-orbit coupling. The octahedral field component produces a splitting of approx. $20,000 \mathrm{~cm}^{-1}$ into an orbital singlet $\left({ }^{4} A_{2 g}\right)$ and two orbital triplets $\left({ }^{4} T_{1 g},{ }^{4} T_{2 g}\right)$. The ${ }^{4} T_{1 g}$ state lies lowest and is further split by spin-orbit coupling into six Kramers doublets. The isotropic $g$-value of the lowest Kramers doublet is given approximately by [10]

$$
g=(2 / 3) x+10 / 3-15 \lambda / 20 D q
$$

For an $F$-state ion $\alpha=3 / 2$. If the value of $D q=837 \mathrm{~cm}^{-1}$ obtained from the electronic spectrum of $\mathrm{Co}_{2} \mathrm{P}_{2} \mathrm{~S}_{6}$ is used in eqn (2), the isotropic $\mathrm{g}$-value for octahedrally coordinated $\mathrm{Co}^{2+}$ is estimated to be $\mathrm{g} \simeq 4.49$. The value estimated is in good agreement with the average $g$-value observed $g_{\text {avg }}=4.4$ (see Table 1) and we conclude that the EPR spectra arise from cations with predominately octahedral coordination. The anisotropy of the experimental $g$-values is indicative of a distorted octahedral $\mathrm{Co}^{2+}$ coordination. In distorded octahedral crystal fields, the $g$-tensor may become highly anisotropic and depend in a complex fashion on the details of the distortion. In the following, we employ a simple crystal field (point charge) model to describe the $\mathrm{Co}^{2+}$ coordination.

\section{Crystal-field model}

The EPR spectra of $\mathrm{Co}^{2+}$ are usually discussed in terms of the Abragham and Price model [10] in which the trigonally distorted octahedral coordination environment is described in terms of empirical values that are introduced as parameterizations of radial integrals arising from different terms in the spherical harmonic expansion of the crystal field $\left(G \sim\left\langle r^{4}\right\rangle\right.$, $\left.H \sim\left\langle r^{2}\right\rangle, I \sim\left\langle r^{4}\right\rangle\right)$. We have used an alternative parameterization of the trigonally distorted octahedral crystal field in terms of the three separable parameters: the trigonal angle $(\theta)$, the familiar cubic field splitting parameter $(D q)$ and the trigonal field splitting parameter $(C p)$ [11].

In terms of these parameters, the crystal-field potential may be written as,

$$
\begin{aligned}
V_{\text {eryst }}= & 21 \sqrt{\pi / 5} C p\left(3 \cos ^{2} \theta-1\right) Y_{2}^{0} \\
& +3 \sqrt{\pi} D q\left(35 \cos ^{4} \theta-30\right. \\
& \left.+\cos ^{2} \theta+3\right) Y_{4}^{0} \\
& +3 \sqrt{35 \pi}\left(\sin ^{3} \theta-\cos \theta\right)\left(Y_{4}^{3}-Y_{4}^{-3}\right),
\end{aligned}
$$

where the $Y_{l}^{m}$ are Legendre polynomials of order $l$. The complete spin-Hamiltonian is,

$$
H=V_{\text {cryst }}+H_{\text {zeeman }},
$$

where $H_{2 \text { ceman }}$ is the magnetic field interaction given by:

$$
H_{\text {zeeman }}=\beta H \cdot\left(\hat{L}+g_{e} \hat{S}\right)+i \hat{L} \cdot \hat{S} .
$$

Diagonalization of the matrix of the crystal-field Hamiltonian [eqn (4)] yields the energy levels of the ground state free-ion term split by the cubic and trigonal crystal-field components, spin-orbit coupling and the applied magnetic field. As the trigonal field splitting and the spin-orbit coupling are of the same order of magnitude, the Hamiltonian was diagonalized with both terms included.

A ${ }^{4} P$ state also arises from the $d^{3}$ free ion and lies about $15,000 \mathrm{~cm}^{-1}$ above the ground state. The ${ }^{4} P$ state must be included in calculations of the ground state splitting, because of its proximity to the ground state and the presence of matrix elements of the crystal field coupling the ${ }^{4} P$ and the ${ }^{4} F$ terms. Therefore, the crystal-Hamiltonian was diagonalized using the 28 functions of the decoupled basis $\left|L, M_{l}, S, M_{s}\right\rangle$ for the ${ }^{4} F$-state $(L=3, S=3 / 2)$ and the 12 basis functions for the ${ }^{4} P$-state $(L=1, S=3 / 2)$, constructed from one electron $d$-orbital functions. The values of other parameters used in the calculation were $B \quad($ Racah $)=500 \mathrm{~cm}^{-1}$ and $i$ (molecular spin-orbit coupling parameter) $=-178 \mathrm{~cm}^{-1}$. Using the Hamiltonian [eqn (4)], the ground state $g$-value ansiotropy was computed for trigonal angle values $50<\theta<58$ and $2<C p / D q<12$, corresponding to the full range of physically reasonable values of these parameters. The relationship between the $g$-value anisotropy $\Delta g=\left(g_{\|}-g_{1}\right)$ and the parameters $\theta$ and $C p / D q$ is displayed as contour plots in Figs 6 and 7 for two values of the crystal field splitting.

The results of the calculation demonstrate that when $C_{p} / D q<8$, the sign of the $g$-value anisotropy depends upon the sign of the trigonal distortion. When $\theta<54.7^{\circ}$ (trigonal elongation) $g_{\|}<g_{\perp}$ and when $\theta>54.7^{\circ}$ (trigonal compression) $g_{\|}>g_{\perp}$. The predicted behaviour is observed experimentally for $\mathrm{Co}^{2+}$ in a variety of trigonally distorted sites 


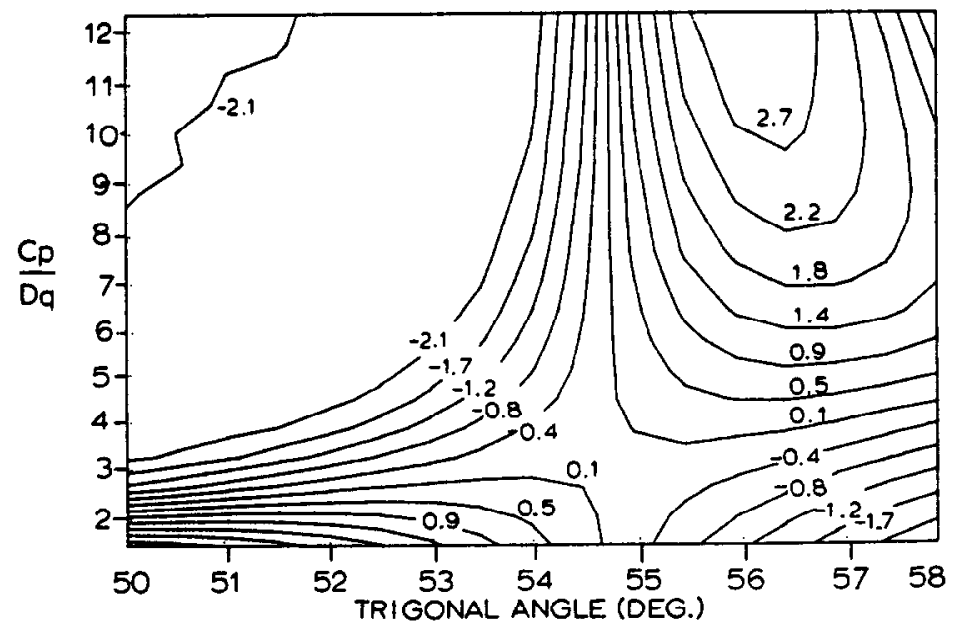

Fig. 6. $g$-Value anisotropy for $\mathrm{Co}^{2+}$ in a trigonally distorted octahedral coordination. The anisotropy $\Delta g=g_{\|}-g_{\perp}$ is shown as a contour plot as a function of the parameters $C p / D q$ and $\theta$. Dq value held constant at $837 \mathrm{~cm}^{-1}$.

(see Table 1). When $C_{p} / D q$ takes larger values ( $>8$ ) the dependence of the anisotropy upon the distortion angle becomes complex and the anisotropy alone cannot be used to determine the sign of the trigonal distortion.

The $\mathrm{Co}^{2+}$ sites in $\mathrm{Cd}_{2} \mathrm{P}_{2} \mathrm{~S}_{6}$ exhibit a $g$-value anisotropy $\Delta g>+1$. The results are unexpected, since in the pure $\mathrm{M}_{2} \mathrm{P}_{2} \mathrm{~S}_{6}$ lattices the metal coordination is trigonally elongated by about $2-3^{\circ}$ [12]. Although antiferromagnetic alignment below $T_{N}=20 \mathrm{~K}$ prevents an experimental determination of the $g$-value anisotropy in $\mathrm{Co}_{2} \mathrm{P}_{2} \mathrm{~S}_{6}$, the calculated value is -2.33 , using parameter values obtained from infrared measurements of the trigonal splitting and $\mathrm{X}$-ray diffraction [13] $\left(C p / D q=6, \theta=51.3^{\circ}\right)$.

Examination of the contour plots of the $g$-value anisotropy (Figs 6 and 7) reveals that $\Delta g=+1$ is consistent with either $C p / D q<2$ and $\theta<54^{\circ}$, or a $C p / D q>5$ and $\theta>55^{\circ}$. Thus, either $C p / D q$ decreases dramatically from its value in $\mathrm{Co}_{2} \mathrm{P}_{2} \mathrm{~S}_{6}$ or the trigonal distortion changes sign. Insertion of a smaller cobalt ion into a cadmium vacancy (or into the VWG) results in a greater $\mathrm{Co}-\mathrm{S}$ bond length than in $\mathrm{Co}_{2} \mathrm{P}_{2} \mathrm{~S}_{6}$. Both $D q$ and $C p$ decrease with increasing bond length, but they do not decrease at the same rate. The magnitude of the $D q$ parameter is proportional to the fourth-order radial one-electron integral and is given by,

$$
D q=\frac{Z e^{2}}{6 a_{0}^{5}}\left\langle r^{4}\right\rangle
$$

where $a_{0}$ is the Bohr radius. $C_{p}$ parametrizes secondorder radial integrals of the form $\left\langle r^{2}\right\rangle$ and is given by,

$$
C p=\frac{2 Z e^{2}}{7 a_{0}^{3}}\left\langle r^{2}\right\rangle \text {. }
$$

Since $D q$ decreases more rapidly than $C p$ with increasing bond length, the behavior expected is for

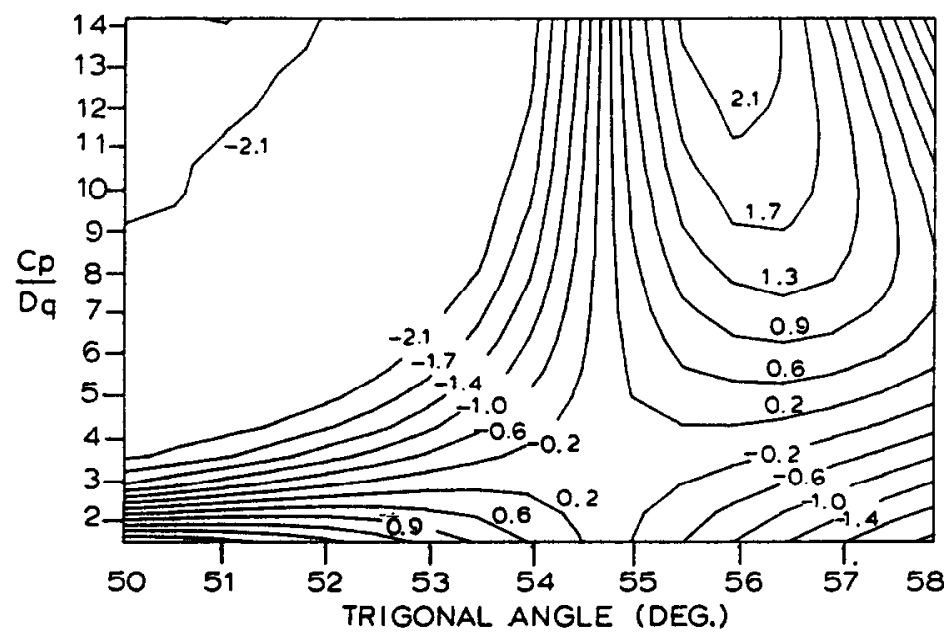

Fig. 7. $g$-Value anisotropy for $\mathrm{Co}^{2+}$ in a trigonally distorted octahedral coordination. The anisotropy $\Delta g=g_{\|}-g \perp$ is shown as a contour plot as a function of the parameters $C_{p} / D q$ and $\theta . D q$ value held constant at $700 \mathrm{~cm}^{-1}$ 
$C p / D q$ to increase as the bond length increases. We conclude that the experimental $g$-value anisotropy is consistent with $\mathrm{Co}^{2+}$ located in trigonally compressed octahedral sites.

The $\Delta g=+1$ contour in Figs 6 and 7 requires a trigonal compression of at least $1^{\circ}$ from octahedral coordination. The replacement of the larger $\mathrm{Cd}^{2+}$ ion (ionic radius $=0.97 \AA$ ) by the smaller $\mathrm{Co}^{2+}$ ion (ionic radius $=0.72 \AA$ ) without local lattice relaxation, leads to an increase in the trigonal angle $\left(51.8^{\circ}\right)$ by $<1^{\circ}$. In order to account for a $4^{\circ}$ elongation from the original trigonal angle, considerable local lattice distortion about the $\mathrm{Co}^{2+}$ must occur. It has been noted previously [14], that the $\mathrm{P}_{2} \mathrm{~S}_{6}^{4-}$ groups exhibit a high degree of flexibility, allowing the first row $\mathrm{M}_{2} \mathrm{P}_{2} \mathrm{~S}_{6}$ materials to maintain essentially unchanged metal coordination geometry although the ionic radius varies substantially. This accommodation is achieved in the main part by variation of the $P-P$ bond length and it is likely that this is the source of the local deformation of the $\mathrm{Co}^{2+}$ coordination environment in $\mathrm{Cd}_{2} \mathrm{P}_{2} \mathrm{~S}_{6}$.

The magnitude of the atomic mean-square vibrational amplitudes in some $M_{2} P_{2} S_{6}$ phases suggests that there is a static disordering of the $\mathrm{MS}_{6}$ octahedra. It has been suggested that this may arise through the existence of energetically equivalent minima for several $\mathbf{M S}_{6}$ configurations [15]. The multi-site EPR spectrum of $\mathrm{Co}^{2+}$ exchanged $\mathrm{Cd}_{2} \mathrm{P}_{2} \mathrm{~S}_{6}$ crystals indicates the presence of at least two nearly octahedral coordination environments. Since the site EPR spectra are similar to that of $\mathrm{Co}^{2+}$ ions occupying $\mathrm{Cd}^{2+}$ vacancies, it seems likely that the exchanged $\mathrm{Co}^{2+}$ also occupy $\mathrm{Cd}^{2+}$ vacancies. However, the low-temperature site interconversion indicates the presence of multiple potential minima for this site. The site responsible for the $4 \mathrm{~K}$, broad EPR spectrum is evidently more labile since it is possible to effect its complete removal by exchanging the lattice with $\mathrm{K}^{+}$while leaving the second site spectrum undiminished (see Fig. 4).

Effect of solvent on the cation exchange process

We have studied the cation exchange reaction of $\mathrm{Cd}_{2} \mathrm{P}_{2} \mathrm{~S}_{6}$ using different paramagnetic ions
$\left(\mathrm{M}^{2+}=\mathrm{Co}^{2+}, \mathrm{Mn}^{2+}, \mathrm{Ni}^{2+}\right)$ and two solvents (pyridine/ $\mathrm{H}_{2} \mathrm{O}$ and $\mathrm{H}_{2} \mathrm{O}$ ). The solvent system may play a significant role through the effect of solvation on the following schematic cation exchange equilibrium process,

$$
\begin{aligned}
& \mathrm{Cd}_{2} \mathrm{P}_{2} \mathrm{~S}_{6} \text { (cryst.) }+x\left(\mathrm{M}^{2+}\right)_{\text {solv }} \\
& \left.<===>\mathrm{Cd}_{(2-x)} \mathrm{M}_{x} \mathrm{P}_{2} \mathrm{~S}_{6} \text { (cryst. }\right)+x\left(\mathrm{Cd}^{2+}\right)_{\text {solv }}
\end{aligned}
$$

or equivalently, for the potassium exchanged host lattice,

$$
\begin{aligned}
& \mathrm{Cd}_{2-x} \mathrm{~K}_{2 x} \mathrm{P}_{2} \mathrm{~S}_{6} \text { (cryst.) }+x\left(\mathrm{M}^{2+}\right)_{\text {solv }} \\
& \left.<===>\mathrm{Cd}_{(2-x)} \mathrm{M}_{x} \mathrm{P}_{2} \mathrm{~S}_{6} \text { (cryst. }\right)+2 x\left(\mathrm{~K}^{+}\right)_{\text {solv }} .
\end{aligned}
$$

The exchange of diamagnetic $\mathrm{Cd}^{2}+$ for paramagnetic $\mathrm{M}^{2+}$ located at the lattice cation vacancy site was followed by EPR. The results of these measurements are summarized in Table 2 and indicate that the stability constants of the solvated ions and the crystal field stabilization energies of the lattice ions exert comparable effects on the final composition of the ion exchanged lattice. The crystal field stabilization energies are:

$$
\mathrm{Mn}^{2+}(0)=\mathrm{Cd}^{2+}(0)<\mathrm{Co}^{2+}(8 D q)<\mathrm{Ni}^{2+}(12 D q) .
$$

The $\mathrm{M}(\mathrm{py})^{2+}$ stability constants at $25^{\circ} \mathrm{C}$ are [16]:

$$
\mathrm{Mn}^{2+}(1.38)<\mathrm{Co}^{2+}(13.8)<\mathrm{Cd}^{2+}(18.6)<\mathrm{Ni}^{2+}(60.3)
$$

On the basis of the crystal field stabilization, $\mathrm{Co}^{2+}$ should enter the lattice vacancies more readily than $\mathrm{Mn}^{2+}$; but, in the presence of pyridine the reverse is found, which we may understand in terms of the equilibria involving the solvent. The pyridine complex of $\mathrm{Mn}^{2+}$ is much less stable than that of $\mathrm{Co}^{2+}$, with the result that manganese occupies the lattice sites much more easily than cobalt when pyridine is used in the solvent medium. When, $\mathrm{Mn}^{2+}$ is exchanged with $\mathrm{Cd}_{2-x} \mathrm{~K}_{2 x} \mathrm{P}_{2} \mathrm{~S}_{6}$ in aqueous medium, the solvated ion enters the VWG but does not enter lattice vacancies until the solvent is removed by

Table 2 .

\begin{tabular}{llll}
\hline Lattice & Ion & Solvent & \multicolumn{1}{c}{ EPR indication } \\
\hline $\mathrm{Cd}_{2} \mathrm{P}_{2} \mathrm{~S}_{6}$ & $\mathrm{Ni}^{2+}$ & $\mathrm{H}_{2} \mathrm{O}$ & No exchange \\
& $\mathrm{Co}^{2+}$ & $\mathrm{H}_{2} \mathrm{O}$ & No exchange \\
& $\mathrm{Mn}^{2+}$ & $\mathrm{H}_{2} \mathrm{O}$ & Rapid exchange at $\mathrm{RT}$ \\
& $\mathrm{Ni}^{2+}$ & $\mathrm{Pyridine}$ & No exchange \\
& $\mathrm{Co}^{2+}$ & Pyridine & Slight exchange \\
& $\mathrm{Mn}^{2+}$ & Pyridine & Rapid exchange at $\mathrm{RT}$ \\
$\mathrm{Cd}_{2-x} \mathrm{~K}_{2 x} \mathrm{P}_{2} \mathrm{~S}_{6}$ & $\mathrm{Ni}^{2+}$ & $\mathrm{H}, \mathrm{O}$ & No exchange \\
& $\mathrm{Co}^{2+}$ & $\mathrm{H}, \mathrm{O}$ & Rapid exchange at $\mathrm{RT}$ \\
& $\mathrm{Mn}^{2+}$ & $\mathrm{H}_{2} \mathrm{O}$ & With heating to remove solvent \\
& $\mathrm{Ni}^{2+}$ & Pyridine & No exchange \\
& $\mathrm{Co}^{2+}$ & Pyridine & No exchange \\
& $\mathrm{Mn}^{2+}$ & Pyridine & With heating to remove solvent \\
\hline
\end{tabular}


heating. The large difference in the solvation energy may account for the ability to exchange $\mathrm{Cd}^{2+}$ for $\mathrm{Mn}^{2+}$ in pyridine solution without pre-exchange with $\mathrm{K}^{+}[6]$. This conclusion is supported by the observation that $\mathrm{K}^{+}$pre-exchange does not assist the insertion of $\mathrm{Mn}^{2+}$ into $\mathrm{Cd}^{2+}$ vacancies, but actually hinders it. $\mathrm{Ni}^{2+}$ is more strongly coordinated by pyridine than $\mathrm{Cd}^{2+}$ and will not exchange despite its large crystal field stabilization energy. Thus, solvation may reverse the effect of crystal field stabilization.

Acknowledgements-We would like to thank the Binational Fulbright Commission for a Fulbright Senior Scholar award (MAH). This research was partially supported by grants from the National Science Foundation (DMR 8818371) and the Donors of the American Chemical Society-Petroleum Research Fund.

\section{REFERENCES}

1. Klingen W., Otto R. and Hahn H., Z. anorg. allg. Chem. 396, 271 (1973); Klingen W., Eulenberger G. and Hahn H., $Z$ anorg. allg. Chem. 401, 97 (1973); Wilson J. A., DiSalvo F. J. and Mahajan S., Adv. Phys. 24, 117 (1975).

2. Lifshitz E., Gentry A. and Francis A. H., J. phys. Chem. 88, 3038 (1984); Cleary D. A. and Francis A. H., J. phys. Chem. 89, 97 (1985); Foot P. J. S. and Shaker N. G., Mater. Res. Bull. 18, 173 (1983);
Yamanaka S. and Hattor M., Nippon Kagaku Kaishi 2, 316 (1983).

3. Clement R., J. chem. Soc. chem. Commun. 647 (1980); Clement R., J. Am. chem. Soc. 103, 6998 (1981).

4. Clement R. and Michalowicz A., Rev. Chim. Miner. 21, 426 (1984).

5. Clement R., Garnier $O$. and Jegoudez J., J. inorg. Chem. 25, 1404 (1986).

6. Cleary D. A., Groh J., Lifshitz E. and Francis A. H., J. phys. Chem. 92, 551 (1988).

7. Taylor B. E., Steger J. and Wold A., J. Solid St. Chem. 7, 461 (1973).

8. Long G. T. and Cleary D. A., J. Phys. C: Condens. Mat. 2, 4747 (1990).

9. Grimshaw J. M. and Read M. G., J. Phys. C: Solid St. Phys. 16, 7053 (1983).

10. Abragam A. and Pryce M. H. L., Proc. R. Soc. A 173, 206 (1951).

11. Gerloch M. and Slade R. C., Ligand-Field Parameters. Cambridge University Press, London (1973).

12. Ouvrard G., Brec R. and Rouxel J., Mater. Res. Bull. 20, 1181 (1985).

13. Nagasundaram N. and Francis A. H., J. Phys. Chem. Solids 50, 163 (1989).

14. Brec R., Solid St. Ionics 22, 3 (1986).

15. Barj M., Lucazeau G., Ouvrard G. and Brec R., Eur. J. Solid St. inorg. Chem. 25, 449 (1988).

16. Bjerrum J., Chem. Rev. 46, 381 (1950); Ablov A. V. and Nazaroda L. V., Z. neorg Khim. 2, 53 (1957); Bjerrum J., Acta chem. Scand. 27, 970 (1973); Howard G. D. and Marianelli R. S., Inorg. Chem. 9, 1738 (1970).

17. Low W., Phys. Rev. 1098, 256 (1958).

18. Low W. and Rubens R. S., Phys. Lett. 1, 316 (1962). 